\section{FRI0553 CANAKINUMAB AS A FIRST-LINE AND SECOND-LINE BIOLOGIC FOR TREATMENT OF SYSTEMIC JUVENILE IDIOPATHIC ARTHRITIS IN CHILDREN UNDER 4 YEARS OF AGE}

Ekaterina Alexeeva ${ }^{1,2}$, Tatyana Dvoryakovskaya ${ }^{1,2}$, Ksenia Isaeva $^{1}$,

Rina Denisova ${ }^{1}$, Margarita Soloshenko ${ }^{1}$, Anna Fetisova ${ }^{1}$, Anna Mamutova ${ }^{1}$, Maria Rudnitskaya ${ }^{1}$, Dariya Vankova ${ }^{1}$, Ivan Kriulin ${ }^{1}$, Kristina Chibisova $^{1}$, Alina Alshevskaya ${ }^{3}$, Andrey Moskalev ${ }^{3} .{ }^{1}$ National Medical Research Center of Children's Health, Moscow, Russian Federation; ${ }^{2}$ I.M. Sechenov First Moscow State Medical University, Moscow, Russian Federation; ${ }^{3}$ Biostatistics and Clinical Trials Center, Novosibirsk, Russian Federation

Background: Development of biologics and their launching into clinical practice has yielded significant progress in pediatric rheumatology. Nevertheless, treatment of severe disorders, and systemic JIA in particular, still remains a challenge. JIA often has the early-onset form. If its course is aggressive and persistent, patients exhibit poor response to treatment with first-line biologics. Effectiveness of the second-line and subsequent biologics, as well as the optimal sequence of prescribing drugs belonging to different classes to children younger than 4 years, remains an open question

Objectives: To compare the efficacy of canakinumab (CAN) in children with SJIA younger than 4 years (in biologic-naïve patients and when prescribed as a second-line biologic).

Methods: The study was conducted as a subanalysis of the prospective cohort study to evaluate the efficacy of biologics in children with sJIA. Comparative analysis involved 17 patients who had initiated CAN treatment at the National Medical Research Center of Children's Health (Moscow, Russia) when aged $<4$ years (9 biologic-naïve patients (the naïve group) and 8 patients switched from tocilizumab therapy (the switched group)). Treatment efficacy was evaluated according to the dynamics of clinical and laboratory signs using the ACRPedi criteria. The Wallace criteria were used to evaluate whether or not remission had been achieved. Treatment safety was evaluated according to the data presented in the Adverse Event Reports

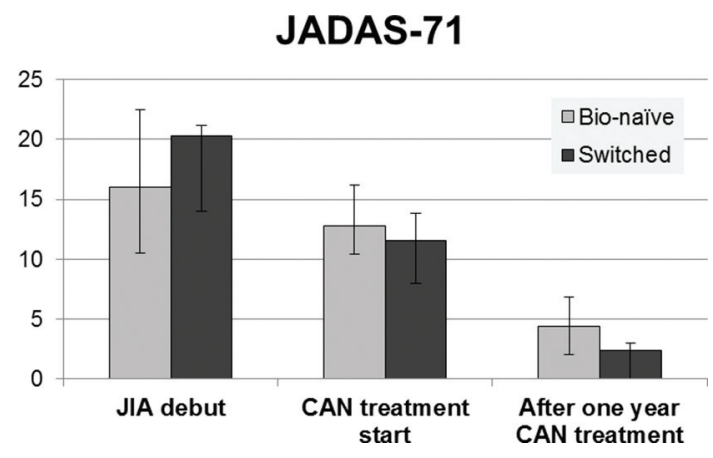

Results: At baseline, patients had comparable duration and severity of the disease. The biologic proved efficacious already after 4 weeks of treatment. Five $(55.6 \%)$ patients in the naïve group and $4(50 \%)$ patients in the switched group achieved ACR90 ( $p>0.999)$ within one year of treatment. A state of inactive disease according to the Wallace criteria was achieved in one patient in each group, $(12.5 \%$ and $11.1 \%$, respectively ( $p>0.999$ ). JADAS-71 decreased significantly from 12.8 (IQR 10.416.2) to 4.4 (IQR 2-6.8) in the naïve group $(p=0.012)$ and from 11.6 (IQR 8-13.88) to 2.35 (IQR 1-3.03) in the switched group ( $\mathrm{p}=0.017)$.

Conclusion: CAN was found to be highly efficacious and have a good safety profile in children younger than 4 years regardless of prior biologic treatment.

Disclosure of Interests: : None declared

DOI: 10.1136/annrheumdis-2019-eular.6640

\section{FRI0554 \\ DNASE1L3 VARIANT IN HYPOCOMPLEMENTEMIC URTICARIAL VASCULITIS SYNDROME IDENTIFIES A DIFFERENT CLINICAL PHENOTYPE}

Marco Ranalli ${ }^{1}$, Chiara Passarelli ${ }^{2}$, Virginia Messia ${ }^{3}$, Manuela Pardeo ${ }^{3}$,

Emanuela $\mathrm{Sacco}^{3}$, Antonella Insalaco ${ }^{3}$, Marina Vivarelli ${ }^{4}$, Fabrizio De Benedetti ${ }^{3}$, Claudia Bracaglia ${ }^{3} .{ }^{1}$ La Sapienza University of Rome, Pediatric Department, Rome, Italy; IRCCS Ospedale Pediatrico Bambino Gesù, Unit of Laboratory of Medical Genetics, Rome, Italy, ${ }^{3}$ IRCCS Ospedale Pediatrico Bambino Gesù, Division of Rheumatology, Rome, Italy; ${ }^{4}$ IRCCS Ospedale Pediatrico Bambino Gesù, Division of Nephrology, Rome, Italy

Background: Hypocomplementemic urticarial vasculitis syndrome (HUVS) is a rare disease characterized by persistent urticarial lesions and hypocomplementemia associated with systemic features involving musculoskeletal, pulmonary, renal and gastrointestinal systems. Systemic lupus erythematosus (SLE) develops in $>50 \%$ of patients with HUVS, although the pathogenesis is unknown.

Objectives: We describe 6 paediatric patients with HUVS, three of whom carry a homozygous variant of DNASE1L3 and present a peculiar clinical phenotype.

Methods: A Targeted Resequencing using a panel including genes already known to be mainly associated to Interferonopathies Lupus-like (DNASE1, DNASE2, DNASE1L3, TREX1) on the Illumina NextSeq ${ }^{\circledR}$ platform was performed. All variants identified were confirmed by Sanger sequencing and, when possible, family members were tested to study the segregation of identified variants. We applied in silico studies only to variants with an allelic frequency $\leq 1 \%$.

Results: All patients described are Caucasian and 3 of them are female. Two patients presented at onset with extended cutaneous manifestation, joints and abdominal involvement with cholecystitis. They did not develop renal or pulmonary involvement. In contrast, the other four patients presented a more severe disease. All of them developed renal involvement (from microhaematuria up to nephrotic syndrome) with renal biopsy showing mesangial glomerulonephritis in three patients and pauci-immune glomerulonephritis (ANCA negative) in one. Moreover, two of them developed also pulmonary vasculitis (Table 1). A homozygous DNASE1L3 variant (c.290_291delCA) was identified in three of these patients. All of them were treated with glucocorticoid and dapsone at onset. Cyclophosphamide, mycophenolate mofetil and azathioprine were used in patients with renal involvement. None of them developed SLE.

Table 1. Patients' clinical characteristics

\begin{tabular}{lcccccc}
\hline & Pt 1 & Pt 2 & Pt 3 & Pt 4 & Pt 5 & Pt 6 \\
\hline Age at onset & 9 y 6/ & 3 y 10/ & 9y 5/12 & 14 y 3/ & 3 y 6/12 & 3 y 1/12 \\
& 12 & 12 & & 12 & & \\
Joints & Yes & Yes & Yes & No & Yes & Yes \\
Ocular & No & Yes & No & No & No & No \\
Abdominal & Yes & Yes & Yes & No & Yes & Yes \\
Pulmonary & No & Yes & Yes & No & No & No \\
Renal & Yes & Yes & Yes & Yes & No & No \\
Antibody anti-C1q & + & + & + & + & + & + \\
Anti-dsDNA & absent & absent & absent & absent & absent & absent \\
C3 (nv 90-180 mg/ & 57 & 63 & 52 & 57 & 19 & 57 \\
dl) & & & & & & \\
C4 (nv 10-40 mg/dl) & 7 & 6 & 2 & 7 & 1 & 1 \\
DNASE1L3 variant & + & + & Ongoing & + & - & - \\
\hline
\end{tabular}

Conclusion: HUVS is very rare disease in childhood. Approximately $50 \%$ of HUVS patients develop SLE. Genetic susceptibility to SLE is recog nized and DNASE1L3-related SLE have been reported. Özçakar et al. have described 5 children from two families with HUVS who carry the same variant on DNASE1L3 that we report here (1). Our patients confirm that variant in DNASE1L3 can cause HUVS and support the hypothesis that this variant is responsible of a more severe phenotype with major organ involvement (renal and pulmonary). Patients with HUVS need to be followed very strictly for the risk to develop SLE. Presence of variant in DNASE1L3 can identify patients with more severe disease and high risk to develop major organ involvement. These patients need more aggressive and possibly life-long immunosuppressive treatment.

\section{REFERENCES:}

[1] Ozçakar ZB, et al. DNASE1L3 Mutations in Hypocomplementemic Urticarial Vasculitis Syndrome. Arthritis Rheum. 2013 Aug;65(8):2183-9.

Disclosure of Interests: Marco Ranalli: None declared, Chiara Passarelli: None declared, Virginia Messia: None declared, Manuela Pardeo: None declared, Emanuela Sacco: None declared, Antonella Insalaco: None 